DOI 10.4171/JEMS/208

Saugata Basu · Dmitrii V. Pasechnik · Marie-Françoise Roy

\title{
Bounding the Betti numbers and computing the Euler-Poincaré characteristic of semi-algebraic sets defined by partly quadratic systems of polynomials
}

Received December 12, 2007 and in revised form June 5, 2008

\begin{abstract}
Let $\mathrm{R}$ be a real closed field, $\mathcal{Q} \subset \mathrm{R}\left[Y_{1}, \ldots, Y_{\ell}, X_{1}, \ldots, X_{k}\right]$, with $\operatorname{deg}_{Y}(Q) \leq 2$, $\operatorname{deg}_{X}(Q) \leq d, Q \in \mathcal{Q}, \#(\mathcal{Q})=m$, and $\mathcal{P} \subset \mathrm{R}\left[X_{1}, \ldots, X_{k}\right]$ with $\operatorname{deg}_{X}(P) \leq d, P \in \mathcal{P}$, $\#(\mathcal{P})=s$, and $S \subset \mathrm{R}^{\ell+k}$ a semi-algebraic set defined by a Boolean formula without negations, with atoms $P=0, P \geq 0, P \leq 0, P \in \mathcal{P} \cup \mathcal{Q}$. We prove that the sum of the Betti numbers of $S$ is bounded by

$$
\ell^{2}(O(s+\ell+m) \ell d)^{k+2 m} .
$$

This is a common generalization of previous results in [4] and [3] on bounding the Betti numbers of closed semi-algebraic sets defined by polynomials of degree $d$ and 2, respectively.

We also describe an algorithm for computing the Euler-Poincaré characteristic of such sets, generalizing similar algorithms described in [4, 9]. The complexity of the algorithm is bounded by $(\ell s m d) O(m(m+k))$.
\end{abstract}

Keywords. Betti numbers, quadratic inequalities, semi-algebraic sets

\section{Introduction and main results}

Let $\mathrm{R}$ be a real closed field and $S \subset \mathrm{R}^{k}$ a semi-algebraic set defined by a Boolean formula with atoms of the form $P>0, P<0, P=0$ for $P \in \mathcal{P} \subset \mathrm{R}\left[X_{1}, \ldots, X_{k}\right]$. We call $S$ a $\mathcal{P}$-semi-algebraic set and the Boolean formula defining $S$ a $\mathcal{P}$-formula. If, instead, the Boolean formula has atoms of the form $P=0, P \geq 0, P \leq 0, P \in \mathcal{P}$, and additionally contains no negation, then we will call $S$ a $\mathcal{P}$-closed semi-algebraic set, and the formula defining $S$ a $\mathcal{P}$-closed formula. Moreover, we call a $\mathcal{P}$-closed semi-algebraic set $S$ basic if the $\mathcal{P}$-closed formula defining $S$ is a conjunction of atoms of the form $P=0, P \geq 0$, $P \leq 0, P \in \mathcal{P}$.

S. Basu: School of Mathematics, Georgia Institute of Technology, Atlanta, GA 30332, U.S.A.; e-mail: saugata.basu@math.gatech.edu

D. V. Pasechnik: MAS Division, School of Physical and Mathematical Sciences, Nanyang Technological University, 21 Nanyang Link, Singapore 637371; e-mail: dima@ntu.edu.sg M.-F. Roy: IRMAR (URA CNRS 305), Université de Rennes I, Campus de Beaulieu, 35042 Rennes Cedex France; e-mail: marie-francoise.roy@univ-rennes1.fr

Mathematics Subject Classification (2010): Primary 14P10, 14P25; Secondary 68W30 
For any closed semi-algebraic set $X \subset \mathrm{R}^{k}$, and any field of coefficients $\mathrm{K}$, we denote by $b_{i}(X, \mathrm{~K})$ the dimension of the $\mathrm{K}$-vector space, $\mathrm{H}_{i}(X, \mathrm{~K})$, which is the $i$-th homology group of $X$ with coefficients in K. We refer to [12] for the definition of homology in the case of $\mathrm{R}$ being an arbitrary real closed field, not necessarily the field of real numbers, and $\mathrm{K}=\mathbb{Q}$. The definition for a more general $\mathrm{K}$ is similar. We denote by $b(X, \mathrm{~K})$ the sum $\sum_{i \geq 0} b_{i}(X, \mathrm{~K})$. We write $b_{i}(X)$ for $b_{i}(X, \mathbb{Z} / 2 \mathbb{Z})$ and $b(X)$ for $b(X, \mathbb{Z} / 2 \mathbb{Z})$. Note that the mod-2 Betti numbers $b_{i}(X)$ are an upper bound on the Betti numbers $b_{i}(X, \mathbb{Q})$ (as a consequence of the Universal Coefficient Theorem for homology (see [20] for example)).

The following result appeared in [4].

Theorem 1.1 ([4]). For a $\mathcal{P}$-closed semi-algebraic set $S \subset \mathrm{R}^{k}, b(S, \mathrm{~K})$ is bounded by $(O(s d))^{k}$, where $s=\#(\mathcal{P})$ and $d=\max _{P \in \mathcal{P}} \operatorname{deg}(P)$.

It is a generalization of the results due to Oleĭnik and Petrovskiı̆ [22], Thom [24], and Milnor [21] on bounding the Betti numbers of real varieties. It provides an upper bound on the sum of the Betti numbers of $\mathcal{P}$-closed semi-algebraic sets in terms of the number and degrees of the polynomials in $\mathcal{P}$ (see also [14] for a slightly more precise bound, and [17] for an extension of this result to arbitrary semi-algebraic sets with a slight worsening of the bound). Notice that this upper bound has singly exponential dependence on $k$, and this dependence is unavoidable (see Example 1.2 below).

In another direction, a restricted class of semi-algebraic sets-namely, semi-algebraic sets defined by quadratic inequalities - has been considered by several researchers [2, 3, 19, 10]. As in the case of general semi-algebraic sets, the Betti numbers of such sets can be exponentially large in the number of variables, as can be seen in the following example.

Example 1.2. The set $S \subset \mathrm{R}^{\ell}$ defined by

$$
Y_{1}\left(Y_{1}-1\right) \geq 0, \ldots, Y_{\ell}\left(Y_{\ell}-1\right) \geq 0
$$

satisfies $b_{0}(S)=2^{\ell}$.

However, it turns out that for a semi-algebraic set $S \subset \mathrm{R}^{\ell}$ defined by $m$ quadratic inequalities, it is possible to obtain upper bounds on the Betti numbers of $S$ which are polynomial in $\ell$ and exponential only in $m$. The first such result is due to Barvinok [3], who proved the following theorem.

Theorem 1.3 ([3]). Let $S \subset \mathrm{R}^{\ell}$ be defined by $Q_{1} \geq 0, \ldots, Q_{m} \geq 0, \operatorname{deg}\left(Q_{i}\right) \leq 2$, $1 \leq i \leq m$. Then $b(S, \mathrm{~K}) \leq \ell^{O(m)}$.

A tighter bound appears in [10].

Even though Theorem 1.1] of [4] and Theorem 1.3 of [3] are stated and proved in the case $K=\mathbb{Q}$ in the original papers, the proofs can be extended without any difficulty to a general $\mathrm{K}$.

Remark 1.4. Notice that the bound in Theorem 1.3 is polynomial in the dimension $\ell$ for fixed $m$, and this fact depends crucially on the assumption that the degrees of the polynomials $Q_{1}, \ldots, Q_{m}$ are at most two. For instance, the semi-algebraic set defined 
by a single polynomial of degree 4 can have Betti numbers exponentially large in $\ell$, as exhibited by the semi-algebraic subset of $\mathrm{R}^{\ell}$ defined by

$$
\sum_{i=0}^{\ell} Y_{i}^{2}\left(Y_{i}-1\right)^{2} \leq 0 .
$$

The above example illustrates the delicate nature of the bound in Theorem 1.3 since a single inequality of degree 4 is enough to destroy the polynomial nature of the bound. In contrast to this, we show in this paper (see Theorem 1.5 below) that a polynomial bound on the Betti numbers of $S$ continues to hold, even if we allow a few (meaning any constant number) of the variables to occur with degrees larger than two in the polynomials used to describe the set $S$.

We now state the main results of this paper.

\subsection{Bounds on the Betti numbers}

We consider semi-algebraic sets defined by polynomial inequalities, in which the dependence of the polynomials on a subset of the variables is at most quadratic. As a result we obtain common generalizations of the bounds stated in Theorems 1.1 and 1.3 . Given any polynomial $P \in \mathrm{R}\left[X_{1}, \ldots, X_{k}, Y_{1}, \ldots, Y_{\ell}\right]$, we will denote by $\operatorname{deg}_{X}(P)\left(\operatorname{resp} \operatorname{deg}_{Y}(P)\right)$ the total degree of $P$ with respect to the variables $X_{1}, \ldots, X_{k}$ (resp. $\left.Y_{1}, \ldots, Y_{\ell}\right)$.

Notation 1. Throughout the paper we fix a real closed field $\mathrm{R}$, and denote by

- $\mathcal{Q} \subset \mathrm{R}\left[Y_{1}, \ldots, Y_{\ell}, X_{1}, \ldots, X_{k}\right]$ a family of polynomials with

$$
\operatorname{deg}_{Y}(Q) \leq 2, \quad \operatorname{deg}_{X}(Q) \leq d, \quad Q \in \mathcal{Q}, \quad \#(\mathcal{Q})=m,
$$

- $\mathcal{P} \subset \mathrm{R}\left[X_{1}, \ldots, X_{k}\right]$ a family of polynomials with

$$
\operatorname{deg}_{X}(P) \leq d, \quad P \in \mathcal{P}, \quad \#(\mathcal{P})=s .
$$

We prove the following theorem.

Theorem 1.5. Let $S \subset \mathrm{R}^{\ell+k}$ be a $\mathcal{P} \cup \mathcal{Q}$-closed semi-algebraic set. Then

$$
b(S) \leq \ell^{2}(O(s+\ell+m) \ell d)^{k+2 m} .
$$

In particular, for $m \leq \ell$, we have $b(S) \leq \ell^{2}(O(s+\ell) \ell d)^{k+2 m}$.

Notice that Theorem 1.5 can be seen as a common generalization of Theorems 1.1 and 1.3 , in the sense that we recover similar bounds (that is, bounds having the same shape) as in Theorem 1.1 (respectively, Theorem 1.3 by setting $\ell$ and $m$ (respectively, $s, d$ and $k$ ) to $O(1)$. Since we use Theorem 1.1 in the proof of Theorem 1.5 (more precisely in the proof of Theorem 2.3 which is a key step in the proof of Theorem 1.5), our proof does not give a new proof of Theorem 1.1. However, our methods do give a new proof of the known bound on Betti numbers in the quadratic case (Theorem 1.3, and this new proof 
is quite different from those given in [3, 10, 18]. The techniques used in [3, 18, 10] do not appear to generalize easily to the parametrized situation considered in this paper.

Note also that as a special case of Theorem 1.5 we obtain a bound on the sum of the Betti numbers of a semi-algebraic set defined over a quadratic map. Such sets have been considered from an algorithmic point of view in [19], where an efficient algorithm is described for computing sample points in every connected component, as well as testing emptiness, of such sets.

More precisely, we show the following.

Corollary 1.6. Let $Q=\left(Q_{1}, \ldots, Q_{k}\right): \mathrm{R}^{\ell} \rightarrow \mathrm{R}^{k}$ be a map where $Q_{i} \in \mathrm{R}\left[Y_{1}, \ldots, Y_{\ell}\right]$ and $\operatorname{deg}\left(Q_{i}\right) \leq 2$ for each $i$. Let $V \subset \mathrm{R}^{k}$ be a $\mathcal{P}$-closed semi-algebraic set for some family $\mathcal{P} \subset \mathrm{R}\left[X_{1}, \ldots, X_{k}\right]$, with $\#(\mathcal{P})=s$ and $\operatorname{deg}(P) \leq d, P \in \mathcal{P}$. Let $S=Q^{-1}(V)$. Then

$$
b(S) \leq \ell^{2}(O(s+\ell+k) \ell d)^{3 k} .
$$

Remark 1.7. Note that the Morse-theoretic techniques developed in [18] give a possible alternative approach for proving Corollary 1.6.

\subsection{Algorithmic implications}

The algorithmic problem of computing topological invariants of semi-algebraic sets (such as the Betti numbers and Euler-Poincaré characteristic) is very well studied. We refer the reader to a recent survey [5] for a detailed account of the recent progress and open problems in this field.

The techniques developed in this paper for obtaining tight bounds on the Betti numbers of semi-algebraic sets defined by partly quadratic systems of polynomials also pave the way towards designing more efficient algorithms for computing the Euler-Poincaré characteristic as well as the Betti numbers of such sets. These algorithms have better complexity than the ones known before.

Definition 1.8. By complexity of an algorithm we will mean the number of arithmetic operations (including comparisons) performed by the algorithm in $\mathrm{R}$. We refer the reader to [12, Chapter 8] for a discussion of various measures of complexity.

We prove the following theorem.

Theorem 1.9. There exists an algorithm that takes as input the description of a $\mathcal{P} \cup \mathcal{Q}$ closed semi-algebraic set, $S$ (following the same notation as in Theorem 1.5 ) and outputs its Euler-Poincaré characteristic $\chi(S)$. The complexity of this algorithm is bounded by $\left(\ell_{s m d}\right)^{O(m(m+k))}$. In the case when $S$ is a basic closed semi-algebraic set the complexity of the algorithm is $(\ell s m d)^{O(m+k)}$.

The algorithm for computing all the Betti numbers has complexity $(\ell s m d)^{2^{O(m+k)}}$ and is much more technical. We omit its description in this paper. It appears in full detail separately in [11]. 
While the complexity of both the algorithms discussed above is polynomial for fixed $m$ and $k$, the complexity of the algorithm for computing the Euler-Poincaré characteristic is significantly better than that of the algorithm for computing all the Betti numbers.

1.2.1. Significance from the computational complexity theory viewpoint . The problem of computing the Betti numbers of semi-algebraic sets in general is a PSPACE-hard problem. We refer the reader to [6], and the references contained therein, for a detailed discussion of those hardness results. In particular, the problem of computing the Betti numbers of a real algebraic variety defined by a single quartic equation is also PSPACE-hard, and the same is true for semi-algebraic sets defined by many quadratic inequalities. On the other hand, as shown in [6] (see also [7]), the problem of computing the Betti numbers of semi-algebraic sets defined by a constant number of quadratic inequalities is solvable in polynomial time. The results mentioned above indicate that the problem of computing the Betti numbers of semi-algebraic sets defined by a constant number of polynomial inequalities is solvable in polynomial time, even if we allow a small (constant-size) subset of the variables to occur with degrees larger than two in the polynomials defining the given set. Note that such a result is not obtainable directly from the results in [6] by the naive method of replacing the monomials having degrees larger than two by a larger set of quadratic ones (introducing new variables and equations in the process).

For general semi-algebraic sets, the algorithmic problem of computing all the Betti numbers is notoriously difficult and only doubly exponential time algorithm is known for this problem. Recently, singly exponential time algorithms [13, 8] have been found for computing the first few Betti numbers of such sets, but the problem of designing singly exponential time algorithm for computing all the Betti numbers remains open. Singly exponential time algorithm is also known for computing the Euler-Poincaré characteristic of general semi-algebraic sets [4].

The rest of the paper is organized as follows. In Section 2 we prove Theorem 1.5. In Section 3 we describe our algorithm for computing the Euler-Poincaré characteristic of sets defined by partly quadratic systems of polynomials and prove Theorem 1.9

\section{Proof of Theorem 1.5}

One of the main ideas behind our proof of Theorem 1.5 is to parametrize a construction introduced by Agrachev in [1] while studying the topology of sets defined by (purely) quadratic inequalities (that is, without the parameters $X_{1}, \ldots, X_{k}$ in our notation). Agrachev constructs a spectral sequence converging to the cohomology of the set being studied. However, it is assumed that the initial quadratic polynomials are generic. In this paper we do not make any genericity assumptions on our polynomials. In order to prove our main theorem we follow another approach based on infinitesimal deformations which avoids the construction of a spectral sequence as done in [1].

We first need to fix some notation and a few preliminary results needed later in the proof. 


\subsection{Mathematical preliminaries}

\subsubsection{Some notation. For all $a \in R$ we define}

$$
\operatorname{sign}(a)= \begin{cases}0 & \text { if } a=0, \\ 1 & \text { if } a>0, \\ -1 & \text { if } a<0 .\end{cases}
$$

Let $\mathcal{A}$ be a finite subset of $\mathrm{R}\left[X_{1}, \ldots, X_{k}\right]$. A sign condition on $\mathcal{A}$ is an element of $\{0,1,-1\}^{\mathcal{A}}$. The realization of the sign condition $\sigma, \mathcal{R}\left(\sigma, \mathbf{R}^{k}\right)$, is the basic semi-algebraic set

$$
\left\{x \in \mathrm{R}^{k} \mid \bigwedge_{P \in \mathcal{A}} \operatorname{sign}(P(x))=\sigma(P)\right\} .
$$

A weak sign condition on $\mathcal{A}$ is an element of $\{\{0\},\{0,1\},\{0,-1\}\}^{\mathcal{A}}$. The realization of the weak sign condition $\rho, \mathcal{R}\left(\rho, \mathrm{R}^{k}\right)$, is the basic, closed semi-algebraic set

$$
\left\{x \in \mathrm{R}^{k} \mid \bigwedge_{P \in \mathcal{A}} \operatorname{sign}(P(x)) \in \rho(P)\right\} .
$$

We often abbreviate $\mathcal{R}\left(\sigma, \mathrm{R}^{k}\right)$ to $\mathcal{R}(\sigma)$, and we denote by $\operatorname{Sign}(\mathcal{A})$ the set of realizable sign conditions $\operatorname{Sign}(\mathcal{A})=\left\{\sigma \in\{0,1,-1\}^{\mathcal{A}} \mid \mathcal{R}(\sigma) \neq \emptyset\right\}$.

More generally, for any $\mathcal{A} \subset \mathrm{R}\left[X_{1}, \ldots, X_{k}\right]$ and a $\mathcal{A}$-formula $\Phi$, we denote by $\mathcal{R}\left(\Phi, \mathrm{R}^{k}\right)$, or simply $\mathcal{R}(\Phi)$, the semi-algebraic set defined by $\Phi$ in $\mathrm{R}^{k}$.

2.1.2. Use of infinitesimals . Later, we extend the ground field $\mathrm{R}$ by infinitesimal elements. We denote by $R\langle\zeta\rangle$ the real closed field of algebraic Puiseux series in $\zeta$ with coefficients in $\mathrm{R}$ (see [12] for more details). The sign of a Puiseux series in $\mathrm{R}\langle\zeta\rangle$ agrees with the sign of the coefficient of the lowest degree term in $\zeta$. This induces a unique order on $\mathrm{R}\langle\zeta\rangle$ which makes $\zeta$ infinitesimal: $\zeta$ is positive and smaller than any positive element of $\mathrm{R}$. When $a \in \mathrm{R}\langle\zeta\rangle$ is bounded from above and below by some elements of $\mathrm{R}, \lim _{\zeta}(a)$ is the constant term of $a$, obtained by substituting 0 for $\zeta$ in $a$. We denote by $\mathrm{R}\left\langle\zeta_{1}, \ldots, \zeta_{n}\right\rangle$ the field $\mathrm{R}\left\langle\zeta_{1}\right\rangle \cdots\left\langle\zeta_{n}\right\rangle$ and in this case $\zeta_{1}$ is positive and infinitesimally small compared to 1 , and for $1 \leq i \leq n-1, \zeta_{i+1}$ is positive and infinitesimally small compared to $\zeta_{i}$, which we abbreviate by writing $0<\zeta_{n} \ll \cdots \ll \zeta_{1} \ll 1$.

Let $\mathrm{R}^{\prime}$ be a real closed field containing R. Given a semi-algebraic set $S$ in $\mathrm{R}^{k}$, the extension of $S$ to $\mathrm{R}^{\prime}$, denoted $\operatorname{Ext}\left(S, \mathrm{R}^{\prime}\right)$, is the semi-algebraic subset of $\mathrm{R}^{\prime k}$ defined by the same quantifier free formula that defines $S$. The set $\operatorname{Ext}\left(S, \mathrm{R}^{\prime}\right)$ is well defined (i.e. it only depends on the set $S$ and not on the quantifier free formula chosen to describe it). This is an easy consequence of the transfer principle (see for instance [12]).

We will need a few results from algebraic topology, which we state here without proofs, referring the reader to papers where the proofs appear.

2.1.3. Mayer-Vietoris inequalities. The following inequalities are consequences of the Mayer-Vietoris exact sequence. 
Proposition 2.1 (Mayer-Vietoris inequalities). Let the subsets $W_{1}, \ldots, W_{t} \subset \mathrm{R}^{n}$ be all closed. Then for each $i \geq 0$ we have

$$
\begin{aligned}
& b_{i}\left(\bigcup_{1 \leq j \leq t} W_{j}\right) \leq \sum_{J \subset\{1, \ldots, t\}} b_{i-\#(J)+1}\left(\bigcap_{j \in J} W_{j}\right), \\
& b_{i}\left(\bigcap_{1 \leq j \leq t} W_{j}\right) \leq \sum_{J \subset\{1, \ldots, t\}} b_{i+\#(J)-1}\left(\bigcup_{j \in J} W_{j}\right) .
\end{aligned}
$$

Proof. See for instance [12].

2.1.4. Topology of sphere bundles . Given a closed and bounded semi-algebraic set $B$, a semi-algebraic $\ell$-sphere bundle over $B$ is given by a continuous semi-algebraic map $\pi: E \rightarrow B$ such that for each $b \in B, \pi^{-1}(b)$ is homeomorphic to $\mathbf{S}^{\ell}$ (the $\ell$-dimensional unit sphere in $\mathrm{R}^{\ell+1}$ ).

We need the following proposition that relates the Betti numbers of $B$ to those of $E$.

Proposition 2.2. Let $B \subset \mathrm{R}^{k}$ be a closed and bounded semi-algebraic set and let $\pi$ : $E \rightarrow B$ be a semi-algebraic $\ell$-sphere bundle with base $B$. Then

$$
b(E) \leq 2 b(B) .
$$

Proof. For $\ell>0$, the assertion follows from the inequality

$$
P_{E}(t) \leq P_{\mathbf{S}^{\ell}}(t) P_{B}(t),
$$

proved in [16, p. 252, (4.1)], where $P_{X}(t)=\sum_{i>0} b_{i}(X) t^{i}$ denotes the Poincaré polynomial of a topological space $X$, and the inequality holds coefficientwise. The inequality 2.3 holds for the Betti numbers with coefficients in $\mathbb{Q}$ as well.

For $\ell=0$, inequality (2.4) is no longer true for the ordinary Betti numbers, as can be observed from the example of the two-dimensional torus, which is a double cover of the Klein bottle. But inequality $(2.3)$ holds for Betti numbers with $\mathbb{Z} / 2 \mathbb{Z}$-coefficients. This follows from the Leray-Serre spectral sequence of the projection map $\pi$, since the homology with coefficients in a local system in this case is the same as the ordinary homology (an elementary proof is given in [10]).

We now return to the proof of Theorem 1.5 .

\subsection{Homogeneous case}

Notation 2. We denote by

- $\mathcal{Q}^{h}$ the family of polynomials obtained by homogenizing $\mathcal{Q}$ with respect to the variables $Y$, i.e.

$$
\mathcal{Q}^{h}=\left\{Q^{h} \mid Q \in \mathcal{Q}\right\} \subset \mathrm{R}\left[Y_{0}, \ldots, Y_{\ell}, X_{1}, \ldots, X_{k}\right],
$$

where $Q^{h}=Y_{0}^{2} Q\left(Y_{1} / Y_{0}, \ldots, Y_{\ell} / Y_{0}, X_{1}, \ldots, X_{k}\right)$,

- $\Phi$ a formula defining a $\mathcal{P}$-closed semi-algebraic set $V$, 
- $A^{h}$ the semi-algebraic set

$$
A^{h}=\bigcup_{Q \in \mathcal{Q}^{h}}\{(y, x)|| y \mid=1 \wedge Q(y, x) \leq 0 \wedge \Phi(x)\},
$$

- $W^{h}$ the semi-algebraic set

$$
W^{h}=\bigcap_{Q \in \mathcal{Q}^{h}}\{(y, x)|| y \mid=1 \wedge Q(y, x) \leq 0 \wedge \Phi(x)\} .
$$

We are going to prove two theorems:

Theorem 2.3.

$$
b\left(A^{h}\right) \leq \ell^{2}(O((s+\ell+m) \ell d))^{m+k} .
$$

Theorem 2.4.

$$
b\left(W^{h}\right) \leq \ell^{2}(O((s+\ell+m) \ell d))^{m+k} .
$$

We need a few preliminary results. Let

$$
\Omega=\left\{\omega \in \mathrm{R}^{m}|| \omega \mid=1, \omega_{i} \leq 0,1 \leq i \leq m\right\} .
$$

Let $\mathcal{Q}=\left\{Q_{1}, \ldots, Q_{m}\right\}$ and $\mathcal{Q}^{h}=\left\{Q_{1}^{h}, \ldots, Q_{m}^{h}\right\}$. For $\omega \in \Omega$ we denote by $\left\langle\omega, \mathcal{Q}^{h}\right\rangle \in$ $\mathrm{R}\left[Y_{0}, \ldots, Y_{\ell}, X_{1}, \ldots, X_{k}\right]$ the polynomial defined by

$$
\left\langle\omega, \mathcal{Q}^{h}\right\rangle=\sum_{i=1}^{m} \omega_{i} Q_{i}^{h}
$$

For $(\omega, x) \in \Omega \times V$, we denote by $\left\langle\omega, \mathcal{Q}^{h}\right\rangle(\cdot, x)$ the quadratic form in $Y_{0}, \ldots, Y_{\ell}$ obtained from $\left\langle\omega, \mathcal{Q}^{h}\right\rangle$ by specializing $X_{i}=x_{i}, 1 \leq i \leq k$.

Let $B \subset \Omega \times \mathbf{S}^{\ell} \times V$ be the semi-algebraic set defined by

$$
B=\left\{(\omega, y, x) \mid \omega \in \Omega, y \in \mathbf{S}^{\ell}, x \in V,\left\langle\omega, \mathcal{Q}^{h}\right\rangle(y, x) \geq 0\right\} .
$$

We denote by $\varphi_{1}: B \rightarrow F$ and $\varphi_{2}: B \rightarrow \mathbf{S}^{\ell} \times V$ the two projection maps in the diagram below.

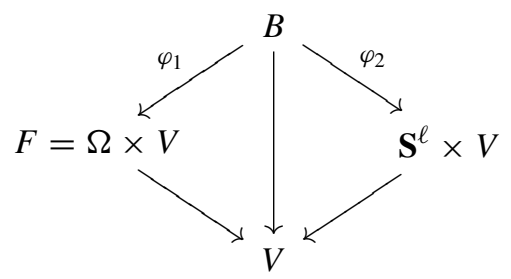

The following key proposition was proved by Agrachev [1] in the unparametrized situation, but as we see below it works in the parametrized case as well. Note that the proposition is quite general and does not require quadratic dependence on the variables $Y$ (i.e. the polynomials $Q_{i}$ need not be quadratic in $Y$ ). Here and in the rest of the paper the phrase 'homotopy equivalence' refers to semi-algebraic homotopy equivalence. 
Proposition 2.5. The semi-algebraic set $B$ is homotopy equivalent to $A^{h}$.

Proof. We first prove that $\varphi_{2}(B)=A^{h}$. If $(y, x) \in A^{h}$, then there exists some $i, 1 \leq i \leq$ $m$, such that $\left(Q_{i}^{h}(y, x) \leq 0\right) \wedge \Phi(x)$. Then for $\omega=\left(-\delta_{1 i}, \ldots,-\delta_{m i}\right)$ (where $\delta_{i j}=1$ if $i=j$, and 0 otherwise), we see that $(\omega, y, x) \in B$. Conversely, if $(y, x) \in \varphi_{2}(B)$, then there exists $\omega \in \Omega$ such that $\left\langle\omega, \mathcal{Q}^{h}\right\rangle(y, x) \geq 0$. Since $\omega \leq 0$ and $\omega \neq 0$, we see that $\left(Q_{i}^{h}(y, x) \leq 0\right) \wedge \Phi(x)$ for some $i, 1 \leq i \leq m$. This shows that $(y, x) \in A^{h}$.

For $(y, x) \in \varphi_{2}(B)$, the fibre

$$
\varphi_{2}^{-1}(y, x)=\left\{(\omega, y, x) \mid \omega \in \Omega,\left\langle\omega, \mathcal{Q}^{h}\right\rangle(y, x) \geq 0\right\},
$$

is a non-empty subset of $\Omega$ defined by a single linear inequality. Thus, each fibre is an intersection of a non-empty closed convex cone with $\mathbf{S}^{m-1}$. The proposition now follows from the well-known Vietoris-Smale theorem [23] since by the above observation each fibre is a closed, bounded and contractible semi-algebraic set.

We will use the following notation.

Notation 3. For a quadratic form $Q \in \mathrm{R}\left[Y_{0}, \ldots, Y_{\ell}\right]$, we denote by index $(Q)$ the number of negative eigenvalues of the symmetric matrix of the corresponding bilinear form, i.e. of the matrix $M$ such that $Q(y)=\langle M y, y\rangle$ for all $y \in \mathrm{R}^{\ell+1}$ (here $\langle\cdot, \cdot\rangle$ denotes the usual inner product). We also denote by $\lambda_{i}(Q), 0 \leq i \leq \ell$, the eigenvalues of $Q$ in non-decreasing order, i.e.

$$
\lambda_{0}(Q) \leq \lambda_{1}(Q) \leq \cdots \leq \lambda_{\ell}(Q)
$$

For $F=\Omega \times V$ as above we denote

$$
F_{j}=\left\{(\omega, x) \in F \mid \operatorname{index}\left(\left\langle\omega, \mathcal{Q}^{h}\right\rangle(\cdot, x)\right) \leq j\right\} .
$$

It is clear that each $F_{j}$ is a closed semi-algebraic subset of $F$ and we get a filtration of the space $F$ given by

$$
F_{0} \subset F_{1} \subset \cdots \subset F_{\ell+1}=F .
$$

Lemma 2.6. The fibre of the map $\varphi_{1}$ over a point $(\omega, x) \in F_{j} \backslash F_{j-1}$ has the homotopy type of a sphere of dimension $\ell-j$.

Proof. Denote by $\lambda_{i}(\omega, x)=\lambda_{i}\left(\left\langle\omega, \mathcal{Q}^{h}\right\rangle(\cdot, x)\right)$ the eigenvalues of $\left\langle\omega, \mathcal{Q}^{h}\right\rangle(\cdot, x)$ in increasing order. First notice that for $(\omega, x) \in F_{j} \backslash F_{j-1}$,

$$
\lambda_{0}(\omega, x) \leq \cdots \leq \lambda_{j-1}(\omega, x)<0 .
$$

Moreover, letting $W_{0}\left(\left\langle\omega, \mathcal{Q}^{h}\right\rangle(\cdot, x)\right), \ldots, W_{\ell}\left(\left\langle\omega, \mathcal{Q}^{h}\right\rangle(\cdot, x)\right)$ be the coordinates with respect to an orthonormal basis consisting of eigenvectors of $\left\langle\omega, \mathcal{Q}^{h}\right\rangle(\cdot, x)$, we see that $\varphi_{1}^{-1}(\omega, x)$ is the subset of $\mathbf{S}^{\ell}=\{\omega\} \times \mathbf{S}^{\ell} \times\{x\}$ defined by

$$
\sum_{i=0}^{\ell} \lambda_{i}(\omega, x) W_{i}\left(\left\langle\omega, \mathcal{Q}^{h}\right\rangle(\cdot, x)\right)^{2} \geq 0, \quad \sum_{i=0}^{\ell} W_{i}\left(\left\langle\omega, \mathcal{Q}^{h}\right\rangle(\cdot, x)\right)^{2}=1 .
$$


Since $\lambda_{i}(\omega, x)<0$ for all $0 \leq i<j$, it follows that for $(\omega, x) \in F_{j} \backslash F_{j-1}$, the fibre $\varphi_{1}^{-1}(\omega, x)$ is homotopy equivalent to the $(k-j)$-dimensional sphere defined by setting

$$
W_{0}\left(\left\langle\omega, \mathcal{Q}^{h}\right\rangle(\cdot, x)\right)=\cdots=W_{j-1}\left(\left\langle\omega, \mathcal{Q}^{h}\right\rangle(\cdot, x)\right)=0
$$

on the sphere defined by

$$
\sum_{i=0}^{\ell} W_{i}\left(\left\langle\omega, \mathcal{Q}^{h}\right\rangle(\cdot, x)\right)^{2}=1 .
$$

For each $(\omega, x) \in F_{j} \backslash F_{j-1}$, let $L_{j}^{+}(\omega, x) \subset \mathrm{R}^{\ell+1}$ denote the sum of the non-negative eigenspaces of $\left\langle\omega, \mathcal{Q}^{h}\right\rangle(\cdot, x)$. Since index $\left(\left\langle\omega, \mathcal{Q}^{h}\right\rangle(\cdot, x)\right)=j$ stays invariant as $(\omega, x)$ varies over $F_{j} \backslash F_{j-1}, L_{j}^{+}(\omega, x)$ varies continuously with $(\omega, x)$.

We denote by $C$ the semi-algebraic set defined as follows. We first define, for $0 \leq$ $j \leq \ell+1$,

$$
C_{j}=\left\{(\omega, y, x)\left|(\omega, x) \in F_{j} \backslash F_{j-1}, y \in L_{j}^{+}(\omega, x),\right| y \mid=1\right\},
$$

and finally we define

$$
C=\bigcup_{j=0}^{\ell+1} C_{j}
$$

The following proposition relates the homotopy type of $B$ to that of $C$.

Proposition 2.7. The semi-algebraic set $C$ defined by (2.13) is homotopy equivalent to $B$.

Before proving the proposition we give an illustrative example.

Example 2.8. In this example $m=2, \ell=3, k=0$, and $\mathcal{Q}^{h}=\left\{Q_{1}^{h}, Q_{2}^{h}\right\}$ with

$$
Q_{1}^{h}=-Y_{0}^{2}-Y_{1}^{2}-Y_{2}^{2}, \quad Q_{2}^{h}=Y_{0}^{2}+2 Y_{1}^{2}+3 Y_{2}^{2} \text {. }
$$

The set $\Omega$ is the part of the unit circle in the third quadrant of the plane, and $F=\Omega$ in this case (since $k=0$ ). In Fig. 1 , we display the fibres of the map $\varphi_{1}^{-1}(\omega) \subset B$ for a sequence of values of $\omega$ starting from $(-1,0)$ and ending with $(0,-1)$. We also show the spheres, $C \cap \varphi_{1}^{-1}(\omega)$, of dimensions 0,1 , and 2 , that these fibres retract to. At $\omega=(-1,0)$, it is easy to verify that index $\left(\left\langle\omega, \mathcal{Q}^{h}\right\rangle\right)=3$, and the fibre $\varphi_{1}^{-1}(\omega) \subset B$ is empty. Starting from $\omega=(-\cos (\arctan (1)),-\sin (\arctan (1)))$ we have index $\left(\left\langle\omega, \mathcal{Q}^{h}\right\rangle\right)=2$, and the fibre $\varphi_{1}^{-1}(\omega)$ is the union of two spherical caps, homotopy equivalent to $\mathbf{S}^{0}$. Starting from
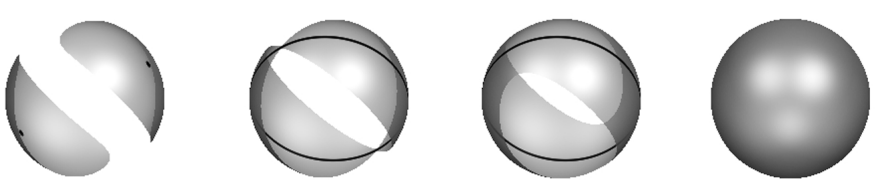

Fig. 1. Type change: $\emptyset \rightarrow \mathbf{S}^{0} \rightarrow \mathbf{S}^{1} \rightarrow \mathbf{S}^{2}$. $\varnothing$ is not shown. 
$\omega=(-\cos (\arctan (1 / 2)),-\sin (\arctan (1 / 2)))$ we have index $\left(\left\langle\omega, \mathcal{Q}^{h}\right\rangle\right)=1$, and the fibre $\varphi_{1}^{-1}(\omega)$ is homotopy equivalent to $\mathbf{S}^{1}$. Finally, starting from $\omega=(-\cos (\arctan (1 / 3))$, $-\sin (\arctan (1 / 3))), \operatorname{index}\left(\left\langle\omega, \mathcal{Q}^{h}\right\rangle\right)=0$, and the fibre $\varphi_{1}^{-1}(\omega)$ stays equal to $\mathbf{S}^{2}$.

Proof of Proposition 2.7. We construct a deformation retraction of $B$ to $C$, as follows. Let

$$
B_{j}=\bigcup_{i=j}^{\ell+1} C_{i} \cup \varphi_{1}^{-1}\left(F_{j-1}\right)
$$

and note that $B_{\ell+1}=B, \ldots, B_{0}=C$.

We construct a sequence of homotopy equivalences from $B_{j+1}$ to $B_{j}$ for every $j=$ $\ell, \ldots, 0$ as follows.

Let $0 \leq j \leq \ell$. For each $(\omega, x) \in F_{j} \backslash F_{j-1}$, we retract the fibre $\varphi_{1}^{-1}(\omega, x)$ to the $(\ell-j)$-dimensional sphere, $L_{j}^{+}(\omega, x) \cap \mathbf{S}^{\ell}$ as follows. Let

$$
W_{0}\left(\left\langle\omega, \mathcal{Q}^{h}\right\rangle(\cdot, x)\right), \ldots, W_{\ell}\left(\left\langle\omega, \mathcal{Q}^{h}\right\rangle(\cdot, x)\right)
$$

be the coordinates with respect to an orthonormal basis consisting of eigenvectors

$$
e_{0}\left(\left\langle\omega, \mathcal{Q}^{h}\right\rangle(\cdot, x)\right), \ldots, e_{\ell}\left(\left\langle\omega, \mathcal{Q}^{h}\right\rangle(\cdot, x)\right)
$$

of $\left\langle\omega, \mathcal{Q}^{h}\right\rangle(\cdot, x)$ corresponding to the non-decreasing sequence of eigenvalues. Then $\varphi_{1}^{-1}(\omega, x)$ is the subset of $\mathbf{S}^{\ell}$ defined by

$$
\sum_{i=0}^{\ell} \lambda_{i}(\omega, x) W_{i}\left(\left\langle\omega, \mathcal{Q}^{h}\right\rangle(\cdot, x)\right)^{2} \geq 0, \quad \sum_{i=0}^{\ell} W_{i}\left(\left\langle\omega, \mathcal{Q}^{h}\right\rangle(\cdot, x)\right)^{2}=1,
$$

and $L_{j}^{+}(\omega, x)$ is defined by $W_{0}\left(\left\langle\omega, \mathcal{Q}^{h}\right\rangle(\cdot, x)\right)=\cdots=W_{j-1}\left(\left\langle\omega, \mathcal{Q}^{h}\right\rangle(\cdot, x)\right)=0$. We re$\operatorname{tract} \varphi_{1}^{-1}(\omega, x)$ to the $(\ell-j)$-dimensional sphere $L_{j}^{+}(\omega, x) \cap \mathbf{S}^{\ell}$ by sending $\left(w_{0}, \ldots, w_{\ell}\right)$ $\in \varphi_{1}^{-1}(\omega, x)$ at time $t$ to $\left(t w_{0}, \ldots, t w_{j-1}, t^{\prime} w_{j}, \ldots, t^{\prime} w_{\ell}\right)$, where $0 \leq t \leq 1$ and

$$
t^{\prime}=\left(\frac{1-t^{2} \sum_{i=0}^{j-1} w_{i}^{2}}{\sum_{i=j}^{\ell} w_{i}^{2}}\right)^{1 / 2}
$$

Notice that even though the local coordinates $W_{0}\left(\left\langle\omega, \mathcal{Q}^{h}\right\rangle(\cdot, x)\right), \ldots, W_{\ell}\left(\left\langle\omega \mathcal{Q}^{h}\right\rangle(\cdot, x)\right)$ in $\mathrm{R}^{\ell+1}$ with respect to the orthonormal basis $\left(e_{0}\left(\left\langle\omega, \mathcal{Q}^{h}\right\rangle(\cdot, x)\right), \ldots, e_{\ell}\left(\left\langle\omega, \mathcal{Q}^{h}\right\rangle(\cdot, x)\right)\right)$ of eigenvectors may not be uniquely defined at the point $(\omega, x)$ (for instance, if the quadratic form $\left\langle\omega, \mathcal{Q}^{h}\right\rangle(\cdot, x)$ has multiple eigenvalues $)$, the retraction is still well defined since it only depends on the decomposition of $\mathrm{R}^{\ell+1}$ into orthogonal complementary subspaces $\operatorname{span}\left(e_{0}, \ldots, e_{j-1}\right)$ and $\operatorname{span}\left(e_{j}, \ldots, e_{\ell}\right)$ which is well defined.

We can thus retract simultaneously all fibres over $F_{j} \backslash F_{j-1}$ continuously, to obtain $B_{j} \subset B$, which is moreover homotopy equivalent to $B_{j+1}$.

Notice that the semi-algebraic set $C_{j}$ is an $\mathbf{S}^{\ell-j}$-bundle over $F_{j} \backslash F_{j-1}$ under the map $\varphi_{1}$, and $C$ is the union of these sphere bundles. Since we have good bounds on the number 
as well as the degrees of polynomials used to define the bases, $F_{j} \backslash F_{j-1}$, we can bound the Betti numbers of each $C_{j}$ using Proposition 2.2. However, the $C_{j}$ could be possibly glued to each other in complicated ways, and thus knowing upper bounds on the Betti numbers of each $C_{j}$ does not immediately produce a bound on the Betti numbers of $C$. In order to get around this difficulty, we consider certain closed subsets $F_{j}^{\prime}$ of $F$, where each $F_{j}^{\prime}$ is an infinitesimal deformation of $F_{j} \backslash F_{j-1}$, and form the base of an $\mathbf{S}^{\ell-j}$-bundle $C_{j}^{\prime}$. Additionally, the $C_{j}^{\prime}$ are glued to each other along sphere bundles over $F_{j}^{\prime} \cap F_{j-1}^{\prime}$, and their union, $C^{\prime}$, is homotopy equivalent to $C$. Since the $C_{j}^{\prime}$ are closed and bounded semi-algebraic sets, and we have good bounds on their Betti numbers as well as the Betti numbers of their non-empty intersections, we can use the Mayer-Vietoris inequalities (Proposition 2.1 to bound the Betti numbers of $C^{\prime}$, which in turn are equal to the Betti numbers of $C$.

We now make precise the argument outlined above.

Let $\Lambda \in \mathrm{R}\left[Z_{1}, \ldots, Z_{m}, X_{1}, \ldots, X_{k}, T\right]$ be the polynomial defined by

$$
\Lambda=\operatorname{det}\left(T \cdot \operatorname{Id}_{\ell+1}-M_{Z \cdot \mathcal{Q}^{h}}\right)=T^{\ell+1}+C_{\ell} T^{\ell}+\cdots+C_{0},
$$

where $Z \cdot \mathcal{Q}^{h}=\sum_{i=1}^{m} Z_{i} Q_{i}^{h}$, and each $C_{i} \in \mathrm{R}\left[Z_{1}, \ldots, Z_{m}, X_{1}, \ldots, X_{k}\right]$.

Note that for $(\omega, x) \in \Omega \times \mathrm{R}^{k}$, the polynomial $\Lambda(\omega, x, T)$, being the characteristic polynomial of a real symmetric matrix, has all its roots real. It then follows from Descartes' rule of signs (see for instance [12]) that for each $(\omega, x) \in \Omega \times \mathrm{R}^{k}$, index $\left(\left\langle\omega, \mathcal{Q}^{h}\right\rangle(\cdot, x)\right)$ is determined by the sign vector

$$
\left(\operatorname{sign}\left(C_{\ell}(\omega, x)\right), \ldots, \operatorname{sign}\left(C_{0}(\omega, x)\right)\right) .
$$

More precisely, the number of sign variations in the sequence

$$
\operatorname{sign}\left(C_{0}(\omega, x)\right), \ldots,(-1)^{i} \operatorname{sign}\left(C_{i}(\omega, x)\right), \ldots,(-1)^{\ell} \operatorname{sign}\left(C_{\ell}(\omega, x)\right),+1
$$

is equal to index $\left(\left\langle\omega, \mathcal{Q}^{h}\right\rangle(\cdot, x)\right)$. Hence, denoting

$$
\mathcal{C}=\left\{C_{0}, \ldots, C_{\ell}\right\} \subset \mathrm{R}\left[Z_{1}, \ldots, Z_{m}, X_{1}, \ldots, X_{k}\right],
$$

we have

Lemma 2.9. $F_{j}$ is the intersection of $F$ with a $\mathcal{C}$-closed semi-algebraic set $D_{j} \subset \mathrm{R}^{m+k}$, for each $0 \leq j \leq \ell+1$.

Notation 4. Let

$$
0<\varepsilon_{0} \ll \cdots \ll \varepsilon_{\ell+1} \ll 1
$$

be infinitesimals. For $0 \leq j \leq \ell+1$, we denote by $\mathrm{R}_{j}$ the field $\mathrm{R}\left\langle\varepsilon_{\ell+1} \ldots \varepsilon_{j}\right\rangle$.

Let

$$
\mathcal{C}_{j}^{\prime}=\left\{P \pm \varepsilon_{j} \mid P \in \mathcal{C}\right\}
$$

Given $\rho \in \operatorname{Sign}(\mathcal{C})$, and $0 \leq j \leq \ell+1$, we denote by $\mathcal{R}\left(\rho_{j}^{c}\right) \subset \mathrm{R}_{j}^{m+k}$ the $\mathcal{C}_{j}^{\prime}$-semialgebraic set defined by the formula $\rho_{j}^{c}$ obtained by taking the conjunction of 
- $-\varepsilon_{j} \leq P \leq \varepsilon_{j}$ for each $P \in \mathcal{C}$ such that $\rho(P)=0$,

- $P \geq-\varepsilon_{j}$ for each $P \in \mathcal{C}$ such that $\rho(P)=1$,

- $P \leq \varepsilon_{j}$ for each $P \in \mathcal{C}$ such that $\rho(P)=-1$.

Similarly, we denote by $\mathcal{R}\left(\rho_{j}^{o}\right) \subset \mathrm{R}_{j}^{m+k}$ the $\mathcal{C}_{j}^{\prime}$-semi-algebraic set defined by the formula $\rho^{o}$ obtained by taking the conjunction of

- $-\varepsilon_{j}<P<\varepsilon_{j}$ for each $P \in \mathcal{C}$ such that $\rho(P)=0$,

- $P>-\varepsilon_{j}$ for each $P \in \mathcal{C}$ such that $\rho(P)=1$,

- $P<\varepsilon_{j}$ for each $P \in \mathcal{C}$ such that $\rho(P)=-1$.

Since the semi-algebraic sets $D_{j}$ defined above in Lemma 2.9 are $\mathcal{C}$-semi-algebraic, each $D_{j}$ is defined by a disjunction of sign conditions on $\mathcal{C}$. More precisely, for each $0 \leq j \leq \ell+1$ let

$$
D_{j}=\bigcup_{\rho \in \Sigma_{j}} \mathcal{R}(\rho)
$$

where $\Sigma_{j} \subset \operatorname{Sign}(\mathcal{C})$.

For each $0 \leq j \leq \ell+1$, let

$$
\begin{aligned}
& D_{j}^{o}=\bigcup_{\rho \in \Sigma_{j}} \mathcal{R}\left(\rho_{j}^{o}\right), \quad D_{j}^{\prime}=\operatorname{Ext}\left(D_{j}^{c}, \mathrm{R}_{j-1}\right) \backslash D_{j-1}^{o}, \\
& D_{j}^{c}=\bigcup_{\rho \in \Sigma_{j}} \mathcal{R}\left(\rho_{j}^{c}\right), \quad F_{j}^{\prime}=\operatorname{Ext}\left(F, \mathrm{R}_{j-1}\right) \cap D_{j}^{\prime},
\end{aligned}
$$

where we set $D_{-1}^{o}=\emptyset$.

Lemma 2.10. For $0 \leq j+1<i \leq \ell+1$,

$$
\operatorname{Ext}\left(D_{i}^{\prime}, \mathrm{R}_{j-1}\right) \cap D_{j}^{\prime}=\emptyset .
$$

Proof. The inclusions

$$
\begin{gathered}
D_{j-1} \subset D_{j} \subset D_{i-1} \subset D_{i}, \\
D_{j-1}^{o} \subset \operatorname{Ext}\left(D_{j}^{c}, \mathrm{R}_{j-1}\right) \subset \operatorname{Ext}\left(D_{i-1}^{o}, \mathrm{R}_{j-1}\right) \subset \operatorname{Ext}\left(D_{i}^{c}, \mathrm{R}_{j-1}\right)
\end{gathered}
$$

follow directly from the definitions of the sets

$$
D_{i}, D_{j}, D_{j-1}, D_{i}^{c}, D_{j}^{c}, D_{i-1}^{o}, D_{j-1}^{o},
$$

and the fact that

$$
\varepsilon_{i} \gg \varepsilon_{i-1} \gg \varepsilon_{j} \gg \varepsilon_{j-1}
$$

It follows immediately that

$$
D_{i}^{\prime}=\operatorname{Ext}\left(D_{i}^{c}, \mathrm{R}_{j-1}\right) \backslash \operatorname{Ext}\left(D_{i-1}^{o}, \mathrm{R}_{j-1}\right)
$$

is disjoint from $\operatorname{Ext}\left(D_{j}^{c}, \mathrm{R}_{j-1}\right)$, and hence also from $D_{j}^{\prime}$.

We now associate to each $F_{j}^{\prime}$ an $\mathbf{S}^{\ell-j}$-bundle as follows. 
For each $(\omega, x) \in F_{j}^{\prime \prime}=\operatorname{Ext}\left(F_{j}, \mathrm{R}_{j-2}\right) \backslash F_{j-1}^{\prime}$, let $L_{j}^{+}(\omega, x) \subset \mathrm{R}_{j-2}^{\ell+1}$ denote the sum of the non-negative eigenspaces of $\left\langle\omega, \mathcal{Q}^{h}\right\rangle(\cdot, x)$ (i.e. $L_{j}^{+}(\omega, x)$ is the largest linear subspace of $\mathrm{R}_{j-2}^{\ell+1}$ on which $\left\langle\omega, \mathcal{Q}^{h}\right\rangle(\cdot, x)$ is positive semi-definite). Since index $\left(\left\langle\omega, \mathcal{Q}^{h}\right\rangle(\cdot, x)\right)=j$ stays invariant as $(\omega, x)$ varies over $F_{j}^{\prime \prime}, L_{j}^{+}(\omega, x)$ varies continuously with $(\omega, x)$.

Let

$$
\lambda_{0}(\omega, x) \leq \cdots \leq \lambda_{j-1}(\omega, x)<0 \leq \lambda_{j}(\omega, x) \leq \cdots \leq \lambda_{\ell}(\omega, x)
$$

be the eigenvalues of $\left\langle\omega, \mathcal{Q}^{h}\right\rangle(\cdot, x)$ for $(\omega, x) \in F_{j}^{\prime \prime}$. There is a continuous extension of the map sending $(\omega, x) \mapsto L_{j}^{+}(\omega, x)$ to $(\omega, x) \in \operatorname{Ext}\left(F_{j}^{\prime}, \mathrm{R}_{j-2}\right)$. To see this observe that for $(\omega, x) \in F_{j}^{\prime \prime}$ the block of the first $j$ (negative) eigenvalues, $\lambda_{0}(\omega, x) \leq \cdots \leq \lambda_{j-1}(\omega, x)$, and hence the sum of the eigenspaces corresponding to them can be extended continuously to any infinitesimal neighbourhood of $F_{j}^{\prime \prime}$, and in particular to $\operatorname{Ext}\left(F_{j}^{\prime}, \mathrm{R}_{j-2}\right)$. Now $L_{j}^{+}(\omega, x)$ is the orthogonal complement of the sum of the eigenspaces corresponding to the block of negative eigenvalues, $\lambda_{0}(\omega, x) \leq \cdots \leq \lambda_{j-1}(\omega, x)$.

We denote by $C_{j}^{\prime} \subset F_{j}^{\prime} \times \mathrm{R}_{j-1}^{\ell+1}$ the semi-algebraic set defined by

$$
C_{j}^{\prime}=\left\{(\omega, y ; x)\left|(\omega, x) \in F_{j}^{\prime}, y \in L_{j}^{+}(\omega, x),\right| y \mid=1\right\} .
$$

Abusing notation, we denote by $\varphi_{1}$ the projection $C_{j}^{\prime} \rightarrow F_{j}^{\prime}$, which makes $C_{j}^{\prime}$ the total space of an $\mathbf{S}^{\ell-j}$-bundle over $F_{j}^{\prime}$.

The following proposition, expressing in precise terms the fact that $C_{j}^{\prime} \cap C_{j-1}^{\prime}$ is an $\mathbf{S}^{\ell-j}$-bundle over $F_{j} \cap F_{j-1}^{\prime}$ under the map $\varphi_{1}$, follows directly from the definition of the sets $C_{j}^{\prime}$ and $F_{j}^{\prime}$.

Proposition 2.11. For every $j$ from $\ell$ to $1, C_{j-1}^{\prime} \cap \operatorname{Ext}\left(C_{j}^{\prime}, \mathrm{R}_{j-2}\right)$ is an $\mathbf{S}^{\ell-j}$-bundle over $\operatorname{Ext}\left(F_{j}^{\prime}, \mathrm{R}_{j-2}\right) \cap F_{j-1}^{\prime}$ under the map $\varphi_{1}$.

We also have the following.

Proposition 2.12. The semi-algebraic set

$$
C^{\prime}=\bigcup_{j=0}^{\ell+1} \operatorname{Ext}\left(C_{j}^{\prime}, \mathrm{R}_{0}\right)
$$

is homotopy equivalent to $\operatorname{Ext}\left(C, \mathrm{R}_{0}\right)$.

Proof. First observe that $C=\lim _{\varepsilon_{\ell+1}} C^{\prime}$ where $C$ is the semi-algebraic set defined in 2.13 above.

Now let

$$
C_{0}=\lim _{\varepsilon_{0}} C^{\prime}, \quad C_{i}=\lim _{\varepsilon_{i}} C_{i-1}, \quad 1 \leq i \leq \ell+1 .
$$

Notice that each $C_{i}$ is a closed and bounded semi-algebraic set. Also, let $C_{i-1, t} \subset \mathrm{R}_{i-1}^{\ell+k}$ be the semi-algebraic set obtained by replacing $\varepsilon_{i}$ in the definition of $C_{i-1}$ by the variable $t$. Then there exists $t_{0}>0$ such that $C_{i-1, t_{1}} \subset C_{i-1, t_{2}}$ for all $0<t_{1}<t_{2} \leq t_{0}$. 
It follows (see [12, Lemma 16.17]) that for each $i, 0 \leq i \leq \ell+1, \operatorname{Ext}\left(C_{i}, \mathrm{R}_{i}\right)$ is homotopy equivalent to $C_{i-1}$ (where $C_{-1}=C^{\prime}$ ).

The proposition is now a consequence of Proposition 2.7.

Proof of Theorem 2.3 In light of Propositions 2.7 and 2.12, it suffices to bound the Betti numbers of the semi-algebraic set $C^{\prime}$. Now,

$$
C^{\prime}=\bigcup_{j=0}^{\ell+1} \operatorname{Ext}\left(C_{j}^{\prime}, \mathrm{R}_{0}\right)
$$

By (2.1) it suffices to bound the Betti numbers of the various intersections amongst the sets $\operatorname{Ext}\left(C_{j}^{\prime}, \mathrm{R}_{0}\right)$ 's. However, by Lemma 2.10 , the only non-empty intersections among $\operatorname{Ext}\left(C_{j}^{\prime}, \mathrm{R}_{0}\right)$ 's are of the form $\operatorname{Ext}\left(C_{j}^{\prime}, \mathrm{R}_{0}\right) \cap \operatorname{Ext}\left(C_{j+1}^{\prime}, \mathrm{R}_{0}\right)$. Using Propositions 2.2 and 2.11 we find that $b\left(C_{j}^{\prime}\right)\left(\operatorname{resp} . b\left(C_{j}^{\prime} \cap C_{j+1}^{\prime}\right)\right)$ is bounded by $2 b\left(F_{j}^{\prime}\right)\left(\operatorname{resp} .2 b\left(\operatorname{Ext}\left(F_{j}^{\prime}, \mathrm{R}_{0}\right) \cap\right.\right.$ $\left.\operatorname{Ext}\left(F_{j+1}^{\prime}, \mathrm{R}_{0}\right)\right)$ ).

Finally, each $F_{j}^{\prime}\left(\right.$ resp. $\left.\operatorname{Ext}\left(F_{j}^{\prime}, \mathrm{R}_{0}\right) \cap \operatorname{Ext}\left(F_{j+1}^{\prime}, \mathrm{R}_{0}\right)\right)$ is a bounded $\mathcal{P}_{j}^{\prime}$-closed semialgebraic set, where $\mathcal{P}_{j}^{\prime}=\mathrm{R}\left[Z_{1}, \ldots, Z_{m}, X_{1}, \ldots, X_{k}\right]$ is defined by

$$
\mathcal{P}_{j}^{\prime}=\mathcal{P} \cup \mathcal{C}_{j}^{\prime} \cup \bigcup_{i=1}^{m}\left\{Z_{i}\right\}
$$

Note that

$$
\begin{aligned}
& \operatorname{deg}(P) \leq \begin{cases}d, & P \in \mathcal{P}, \\
d(\ell+1), & P \in \mathcal{C}_{j}^{\prime},\end{cases} \\
& \#(\mathcal{P})=s, \quad \#\left(\mathcal{C}_{j}^{\prime}\right)=2(\ell+1) .
\end{aligned}
$$

Now applying Theorem 1.1 we obtain

$$
b\left(F_{j}^{\prime}\right), b\left(\operatorname{Ext}\left(F_{j}^{\prime}, \mathrm{R}_{0}\right) \cap \operatorname{Ext}\left(F_{j+1}^{\prime}, \mathrm{R}_{0}\right)\right) \leq(O((s+\ell+m) \ell d))^{m+k} .
$$

Applying Proposition 2.2 and 2.16 we immediately obtain

$$
b\left(C_{j}^{\prime}\right), b\left(\operatorname{Ext}\left(C_{j}^{\prime}, \mathrm{R}_{0}\right) \cap \operatorname{Ext}\left(C_{j+1}^{\prime}, \mathrm{R}_{0}\right)\right) \leq(O((s+\ell+m) \ell d))^{m+k} .
$$

Finally, using inequality 2.1] and Lemma 2.10 we get

$$
b\left(C^{\prime}\right)=b\left(\bigcup_{j=0}^{\ell} C_{j}^{\prime}\right) \leq \ell^{2}(O((s+\ell+m) \ell d))^{m+k} .
$$

The theorem now follows from Propositions 2.12 2.5 and 2.7

Proof of Theorem 2.4 Apply (2.2) together with Theorem 2.3 


\subsection{General case}

We now prove the general version of Theorem 2.3. We follow Notation 2

Theorem 2.13. Let $W \subset \mathrm{R}^{\ell} \times \mathrm{R}^{k}$ be a semi-algebraic set defined by

$$
W=\bigcap_{Q \in \mathcal{Q}}\{(y, x) \mid Q(y, x) \leq 0 \wedge \Phi(x)\},
$$

where $\Phi(x)$ is a $\mathcal{P}$-closed formula defining a bounded $\mathcal{P}$-closed semi-algebraic set $V$ $\subset \mathrm{R}^{k}$. Then

$$
b(W) \leq \ell^{2}(O((s+\ell+m) \ell d))^{m+k} .
$$

Proof. Let $1 \gg \varepsilon>0$ be an infinitesimal and let $B_{\ell}(0,1 / \varepsilon)$ denote the closed ball in $\mathrm{R}\langle\varepsilon\rangle^{\ell}$ centred at the origin and of radius $1 / \varepsilon$.

Let $W_{\varepsilon} \subset \mathrm{R}^{\ell+k}$ be the set defined by

$$
W_{\varepsilon}=W \cap\left(B_{\ell}(0,1 / \varepsilon) \times \mathrm{R}^{k}\right) .
$$

It follows from the local conical structure of semi-algebraic sets at infinity [15, Theorem 9.3.6] that $W_{\varepsilon}$ has the same homotopy type as $\operatorname{Ext}(W, \mathrm{R}\langle\varepsilon\rangle)$. Let

$$
Q_{0}=\varepsilon^{2}\left(Y_{1}^{2}+\cdots+Y_{\ell}^{2}\right)-1,
$$

and $W_{\varepsilon}^{h} \subset \mathbf{S}^{\ell} \times \mathrm{R}\langle\varepsilon\rangle^{k}$ be the semi-algebraic set defined by

$$
W_{\varepsilon}^{h}=\bigcap_{i=0}^{m}\left\{(y, x)|| y \mid=1 \wedge Q_{i}^{h}(y, x) \leq 0 \wedge \Phi(x)\right\} .
$$

It is clear that $W_{\varepsilon}^{h}$ is a union of two disjoint, closed and bounded semi-algebraic sets, each homeomorphic to $W_{\varepsilon}$. Hence, for every $i=0, \ldots, k+\ell-1$,

$$
b_{i}\left(W_{\varepsilon}^{h}\right)=2 b_{i}\left(W_{\varepsilon}\right)=2 b_{i}(W) .
$$

The theorem is proved by applying Theorem 2.4 to $W_{\varepsilon}^{h}$.

\subsection{Proof of Theorem 1.5}

We are now in a position to prove Theorem 1.5 We first need a few preliminary results.

Given a list of polynomials $\mathcal{A}=\left\{A_{1}, \ldots, A_{t}\right\}$ with coefficients in R, we introduce $t$ infinitesimals, $1 \gg \delta_{1} \gg \cdots \gg \delta_{t}>0$.

We define $\mathcal{A}_{>i}=\left\{A_{i+1}, \ldots, A_{t}\right\}$ and

$$
\begin{aligned}
\Sigma_{i} & =\left\{A_{i}=0, A_{i}=\delta_{i}, A_{i}=-\delta_{i}, A_{i} \geq 2 \delta_{i}, A_{i} \leq-2 \delta_{i}\right\}, \\
\Sigma_{\leq i} & =\left\{\Psi \mid \Psi=\bigwedge_{j=1, \ldots, i} \Psi_{i}, \Psi_{i} \in \Sigma_{i}\right\} .
\end{aligned}
$$

If now $\Phi$ is any $\mathcal{A}$-closed formula, we denote by $\mathcal{R}_{i}(\Phi)$ the extension of $\mathcal{R}(\Phi)$ to $\mathrm{R}\left\langle\delta_{1}, \ldots, \delta_{i}\right\rangle^{k}$. For $\Psi \in \Sigma_{\leq i}$, we denote by $\mathcal{R}_{i}(\Psi)$ the realization of $\Psi$ and by $b(\Psi)$ the sum of the Betti numbers of $\mathcal{R}_{i}(\Psi)$. 
Proposition 2.14. For every $\mathcal{A}$-closed formula $\Phi$,

$$
b(\Phi) \leq \sum_{\Psi \in \Sigma_{\leq t}} b(\Psi) .
$$

Proof. See [12, Proposition 7.39].

Proof of Theorem 1.5. First note that we can assume (by adding to $\mathcal{Q}$ an extra quadratic inequality if necessary) that the set $S$ is bounded.

Denoting $\mathcal{P}=\left\{P_{1}, \ldots, P_{s}\right\}$, define $\mathcal{B}=\left\{B_{1}, \ldots, B_{s+m}\right\}$, where

$$
B_{i}= \begin{cases}Q_{i}, & 1 \leq i \leq m, \\ P_{i-m}, & m+1 \leq i \leq m+s .\end{cases}
$$

It follows from Proposition 2.14 that in order to bound $b(S)$, it suffices to bound $b(T)$, where $T$ is defined by

$$
\bigwedge_{i=1}^{s+m} B_{i}^{2}\left(B_{i}^{2}-\delta_{i}^{2}\right)^{2}\left(B_{i}^{2}-4 \delta_{i}^{2}\right) \geq 0
$$

We now introduce $m$ new variables, $Z_{1}, \ldots, Z_{m}$ and let

$$
\mathcal{A}=\left\{A_{1}, \ldots, A_{s+m}\right\} \subset \mathrm{R}\left[Y_{1}, \ldots, Y_{\ell}, X_{1}, \ldots, X_{k}, Z_{1}, \ldots, Z_{m}\right]
$$

be defined by

$$
A_{i}= \begin{cases}Z_{i}, & 1 \leq i \leq m, \\ P_{i-m}, & m+1 \leq i \leq m+s .\end{cases}
$$

Consider the semi-algebraic set $T^{\prime} \subset \mathrm{R}^{m+k+l}$ defined by

$$
\bigwedge_{i=1}^{s+m} A_{i}^{2}\left(A_{i}^{2}-\delta_{i}^{2}\right)^{2}\left(A_{i}^{2}-4 \delta_{i}^{2}\right) \geq 0 \wedge \bigwedge_{i=1}^{m}\left(Z_{i}-Q_{i}=0\right) .
$$

Clearly, $T$ is homeomorphic to $T^{\prime}$. Notice that the number of polynomials in the definition of $T^{\prime}$, which depend only on $X$ and $Z$ is $s+m$, and the degrees of these polynomials are bounded by $6 d$. The number of polynomials depending on $X, Y$ and $Z$ is $m$ and these are of degree at most 2 in $Y$ and at most $d$ in the remaining variables. Thus, we can apply Theorem 2.13 to obtain

$$
b(S) \leq b\left(T^{\prime}\right) \leq \ell^{2}(O(s+\ell+m) \ell d)^{k+2 m} .
$$

This proves the theorem.

Proof of Corollary 1.6 Introduce $k$ new variables, $Z_{1}, \ldots, Z_{k}$, and let $\tilde{Q}_{i}=Z_{i}-Q_{i}$ for $1 \leq i \leq k$. Define the semi-algebraic set $\tilde{S} \subset \mathrm{R}^{\ell+k}$ by

$$
\tilde{S}=\left\{(y, x) \mid \bigwedge_{i=1}^{k} \tilde{Q}_{i}(y, x)=0 \wedge \Phi(x)\right\} .
$$

It is clear that $\tilde{S}$ is semi-algebraically homeomorphic to $S$. Applying Theorem 1.5 to $\tilde{S}$, we obtain the desired bound. 


\section{Algorithm for computing the Euler-Poincaré characteristic}

We first need a few preliminary definitions and results.

\subsection{Some algorithmic and mathematical preliminaries}

Recall that for a closed and bounded semi-algebraic set $S \subset \mathrm{R}^{k}$, the Euler-Poincaré characteristic of $S$, denoted by $\chi(S)$, is defined by

$$
\chi(S)=\sum_{i=0}^{k}(-1)^{i} b_{i}(S) .
$$

Moreover, we have the following additivity property which is classical.

Proposition 3.1. Let $X_{1}$ and $X_{2}$ be closed and bounded semi-algebraic sets. Then

$$
\chi\left(X_{1} \cap X_{2}\right)=\chi\left(X_{1}\right)+\chi\left(X_{2}\right)-\chi\left(X_{1} \cup X_{2}\right) .
$$

Recall also that for a locally closed semi-algebraic set $S$, the Borel-Moore Euler-Poincaré characteristic of $S$, denoted by $\chi^{B M}(S)$, is defined by

$$
\chi^{B M}(S)=\sum_{i=0}^{k}(-1)^{i} b_{i}^{B M}(S),
$$

where $b_{i}^{B M}(S)$ denotes the dimension of the $i$-th Borel-Moore homology group $\mathrm{H}_{i}^{B M}(S, \mathbb{Z} / 2 \mathbb{Z})$ of $S$. Note that $\chi^{B M}(S)=\chi(S)$ for $S$ closed and bounded.

Note that $\chi^{B M}(S)$ has the following classically known (see e.g. [12] for a proof) additivity property.

Proposition 3.2. Let $X_{1}$ and $X_{2}$ be locally closed semi-algebraic sets such that $X_{1} \cap X_{2}$ $=\emptyset$. Then

$$
\chi^{B M}\left(X_{1} \cup X_{2}\right)=\chi^{B M}\left(X_{1}\right)+\chi^{B M}\left(X_{2}\right),
$$

provided that $X_{1} \cup X_{2}$ is locally closed as well.

Let $Z \subset \mathrm{R}^{k}$ and $Q \in \mathrm{R}\left[X_{1}, \ldots, X_{k}\right]$. We define

$$
\begin{aligned}
& \mathcal{R}(Q=0, Z)=\{x \in Z \mid Q(x)=0\}, \\
& \mathcal{R}(Q>0, Z)=\{x \in Z \mid Q(x)>0\}, \\
& \mathcal{R}(Q<0, Z)=\{x \in Z \mid Q(x)<0\} .
\end{aligned}
$$

Corollary 3.3. Let $Z \subset \mathrm{R}^{k}$ be a locally closed semi-algebraic set. Then

$$
\chi^{B M}(Z)=\chi^{B M}(\mathcal{R}(Q=0, Z))+\chi^{B M}(\mathcal{R}(Q>0, Z))+\chi^{B M}(\mathcal{R}(Q<0, Z)) .
$$


Notation 5. Let $Z \subset \mathrm{R}^{k}$ be a locally closed semi-algebraic set and let $\mathcal{A}$ be a finite subset of $\mathrm{R}\left[X_{1}, \ldots, X_{k}\right]$. The realization of the sign condition $\rho \in\{0,1,-1\}^{\mathcal{A}}$ on $Z$ is

$$
\mathcal{R}(\rho, Z)=\left\{x \in Z \mid \bigwedge_{A \in \mathcal{A}} \operatorname{sign}(A(x))=\rho(A)\right\},
$$

and its Borel-Moore Euler-Poincaré characteristic is denoted $\chi^{B M}(\rho, Z)$.

We denote by $\operatorname{Sign}(\mathcal{A}, Z)$ the list of $\rho \in\{0,1,-1\}^{\mathcal{A}}$ such that $\mathcal{R}(\rho, Z)$ is non-empty. We denote by $\chi^{B M}(\mathcal{A}, Z)$ the list of the Euler-Poincaré characteristics $\chi^{B M}(\rho, Z)=$ $\chi^{B M}(\mathcal{R}(\rho, Z))$ for $\rho \in \operatorname{Sign}(\mathcal{A}, Z)$.

Finally, given two finite families of polynomials, $\mathcal{A} \subset \mathcal{A}^{\prime}$, and $\rho \in\{0,1,-1\}^{\mathcal{A}}$, $\rho^{\prime} \in\{0,1,-1\}^{\mathcal{A}^{\prime}}$, we define $\rho \prec \rho^{\prime}$ by: $\rho(P)=\rho^{\prime}(P)$ for all $P \in \mathcal{A}$.

We will use the following algorithm for computing the list $\chi^{B M}(\mathcal{A}, Z)$ described in [12]. We recall here the input, output and complexity of the algorithm.

Algorithm 1 (Euler-Poincaré characteristic of sign conditions).

Input: A finite list $\mathcal{A}=\left\{A_{1}, \ldots, A_{t}\right\}$ of polynomials in $\mathrm{R}\left[X_{1}, \ldots, X_{k}\right]$.

Output: The list $\chi^{B M}(\mathcal{A})$.

Complexity analysis. Let $d$ be a bound on the degrees of the polynomials in $\mathcal{A}$, and $t=\#(\mathcal{A})$. The number of arithmetic operations is bounded by

$$
t^{k+1} O(d)^{k}+t^{k}\left(\left(k \log _{2}(s)+k \log _{2}(d)\right) d\right)^{O(k)} .
$$

The algorithm also involves the inversion of matrices of size $t^{k} O(d)^{k}$ with integer coefficients.

\subsection{Algorithms for the Euler-Poincaré characteristic}

We first deal with the special case of polynomials which are homogeneous and of degree two in the variables $Y_{0}, \ldots, Y_{\ell}$, and in this case we describe algorithms (Algorithms 2 and 3 below) for computing the Euler-Poincaré characteristic of the sets $A^{h}$ and $W^{h}$ respectively. We then use Algorithm 3 to derive algorithms for computing the EulerPoincaré characteristic in the general case (Algorithms 4 and 5 below).

\subsubsection{Homogeneous quadratic polynomials .}

Algorithm 2 (Euler-Poincaré characteristic, homogeneous union case).

\section{Input:}

- a family of polynomials, $\mathcal{Q}^{h} \subset \mathrm{R}\left[Y_{0}, \ldots, Y_{\ell}, X_{1}, \ldots, X_{k}\right]$, with $\operatorname{deg}_{Y}(Q) \leq 2$, $\operatorname{deg}_{X}(Q) \leq d, Q \in \mathcal{Q}^{h}, \#\left(\mathcal{Q}^{h}\right)=m$, homogeneous with respect to $Y$,

- another family, $\mathcal{P} \subset \mathrm{R}\left[X_{1}, \ldots, X_{k}\right]$, with $\operatorname{deg}_{X}(P) \leq d, P \in \mathcal{P}, \#(\mathcal{P})=s$,

- a formula $\Phi$ defining a bounded $\mathcal{P}$-closed semi-algebraic set $V$. 
Output: the Euler-Poincare characteristic $\chi\left(A^{h}\right)$, where $A^{h}$ is the semi-algebraic set defined by

$$
A^{h}=\bigcup_{Q \in \mathcal{Q}^{h}}\{(y, x)|| y \mid=1 \wedge Q(y, x) \leq 0 \wedge \Phi(x)\} .
$$

\section{Procedure:}

Step 1. Let $Z=\left(Z_{1}, \ldots, Z_{m}\right)$ be variables and let $M$ be the symmetric matrix with entries in $\mathrm{R}\left[Z_{1}, \ldots, Z_{m}, X_{1}, \ldots, X_{k}\right]$ associated to the quadratic form $\left\langle Z, \mathcal{Q}^{h}\right\rangle$. Obtain $C_{i} \in \mathrm{R}\left[Z_{1}, \ldots, Z_{m}, X_{1}, \ldots, X_{k}\right]$ by computing the determinant

$$
\operatorname{det}\left(T \cdot \operatorname{Id}_{\ell+1}-M\right)=T^{\ell+1}+C_{\ell} T^{\ell}+\cdots+C_{0} .
$$

Step 2. Compute $\chi^{B M}(\mathcal{C}, F)$ as follows. Call Algorithm 1 with input $\mathcal{C}^{\prime}=\mathcal{C} \cup \mathcal{P}$. Compute from the output the list $\chi^{B M}(\mathcal{C}, F)$, using the additivity property of the Borel-Moore Euler-Poincaré characteristic (Proposition 3.2). For each $\rho \in\{0,+1,-1\}^{\mathcal{C}}$ such that there exists $\rho^{\prime} \in \operatorname{Sign}\left(\mathcal{C}^{\prime}, F\right)$ with $\rho \prec \rho^{\prime}$ (see Notation 5) and $\rho^{\prime}\left(Z_{j}\right) \in\{0,-1\}$ for $1 \leq j \leq m$, compute

$$
\chi^{B M}(\rho, F)=\sum_{\substack{\rho^{\prime}, \rho \prec \rho^{\prime}, \rho^{\prime}\left(Z_{j}\right) \in\{0,-1\}, 1 \leq j \leq s}} \chi^{B M}\left(\rho^{\prime}, F\right) .
$$

Step 3. Output

$$
\chi\left(A^{h}\right)=\sum_{\rho \in \operatorname{Sign}(\mathcal{C}, F)} \chi^{B M}(\mathcal{R}(\rho, F)) \cdot\left(1+(-1)^{k-n(\rho)}\right),
$$

where $n(\rho)$ denotes the number of sign variations in the sequence

$$
\rho\left(C_{0}\right), \ldots,(-1)^{i} \rho\left(C_{i}\right), \ldots,(-1)^{\ell} \rho\left(C_{\ell}\right),+1 .
$$

Proof of correctness. It follows from Lemma 2.6 that for any $\rho \in \operatorname{Sign}(\mathcal{C}, F)$,

$$
\chi^{B M}\left(\varphi_{1}^{-1}(\mathcal{R}(\rho))\right)=\chi^{B M}(\mathcal{R}(\rho)) \cdot\left(1+(-1)^{k-n(\rho)}\right) .
$$

Also, by virtue of Proposition 2.5 we have

$$
\chi^{B M}(B)=\chi\left(A^{h}\right), \quad \text { where } \quad B=\bigcup_{\rho \in \operatorname{Sign}(\mathcal{C}, F)} \varphi^{-1}(\mathcal{R}(\rho)) .
$$

The correctness of the algorithm is now a consequence of the additivity property of the Borel-Moore Euler-Poincaré characteristic (Proposition 3.2) and the correctness of Algorithm 1

Complexity analysis. The complexity of the algorithm is $(\ell s m d)^{O(m+k)}$ using the complexity of Algorithm 1

We are now in a position to describe the algorithm for computing the Euler-Poincaré characteristic in the homogeneous intersection case. 
Algorithm 3 (Euler-Poincaré characteristic, homogeneous intersection case).

Input:

- a family of polynomials, $\mathcal{Q}^{h}=\left\{Q_{1}^{h}, \ldots, Q_{m}^{h}\right\} \subset \mathrm{R}\left[Y_{0}, \ldots, Y_{\ell}, X_{1}, \ldots, X_{k}\right]$, with $\operatorname{deg}_{Y}(Q) \leq 2, \operatorname{deg}_{X}(Q) \leq d, Q \in \mathcal{Q}^{h}$, homogeneous with respect to $Y$,

- another family, $\mathcal{P} \subset \mathrm{R}\left[X_{1}, \ldots, X_{k}\right]$, with $\operatorname{deg}_{X}(P) \leq d, P \in \mathcal{P}, \#(\mathcal{P})=s$,

- a formula $\Phi$ defining a bounded $\mathcal{P}$-closed semi-algebraic set $V$.

Output: the Euler-Poincaré characteristic $\chi\left(W^{h}\right)$, where $W^{h}$ is the semi-algebraic set defined by

$$
W^{h}=\bigcap_{Q \in \mathcal{Q}^{h}}\{(y, x)|| y \mid=1 \wedge Q(y, x) \leq 0 \wedge \Phi(x)\} .
$$

\section{Procedure:}

Step 1. For each subset $J \subset[m]$ compute $\chi\left(A^{J}\right)$ using Algorithm 2, where

$$
A^{J}=\bigcup_{Q \in J}\{(y, x)|| y \mid=1 \wedge Q(y, x) \leq 0 \wedge \Phi(x)\} .
$$

Step 2. Output

$$
\chi\left(W^{h}\right)=\sum_{J \subset \mathcal{Q}}(-1)^{\#(J)+1} \chi\left(A^{J}\right) .
$$

Proof of correctness. First note that 3.1) can be easily deduced from Proposition 3.1 by induction. The correctness of the algorithm is now a consequence of the correctness of Algorithm 2 .

Complexity analysis. There are $2^{m}$ calls to Algorithm 2. Using the complexity analysis of Algorithm 2, the complexity of the algorithm is bounded by $(\ell s m d){ }^{O(m+k)}$.

\subsubsection{The case of intersections}

Algorithm 4 (Euler-Poincaré characteristic, intersection case).

\section{Input:}

- a family of polynomials, $\mathcal{Q} \subset \mathrm{R}\left[Y_{1}, \ldots, Y_{\ell}, X_{1}, \ldots, X_{k}\right]$, with $\operatorname{deg}_{Y}(Q) \leq 2, \operatorname{deg}_{X}(Q)$ $\leq d, Q \in \mathcal{Q}, \#(\mathcal{Q})=m$,

- another family of polynomials, $\mathcal{P} \subset \mathrm{R}\left[X_{1}, \ldots, X_{k}\right]$, with $\operatorname{deg}_{X}(Q) \leq d, P \in \mathcal{P}$, $\#(\mathcal{P})=s$,

- a $\mathcal{P}$-closed formula $\Phi$ defining a $\mathcal{P}$-closed semi-algebraic set $V \subset \mathrm{R}^{k}$.

Output: the Euler-Poincaré characteristic $\chi(W)$, where $W$ is the semi-algebraic set defined by

$$
W=\bigcap_{Q \in \mathcal{Q}}\{(y, x) \mid Q(y, x) \leq 0 \wedge \Phi(x)\}
$$

\section{Procedure:}

Step 1. Replace $\mathcal{Q}^{h}$ by $\mathcal{Q}^{h} \cup\left\{Q_{0}^{h}\right\}$ with $Q_{0}=\varepsilon^{2}\left(Y_{1}^{2}+\cdots+Y_{\ell}^{2}\right)-1$. Define

$$
W_{\varepsilon}^{h}=\bigcap_{Q^{h} \in \mathcal{Q}^{h}}\left\{(y, x)|| y \mid=1 \wedge Q^{h}(y, x) \leq 0 \wedge \Phi(x)\right\} .
$$


Step 2. Using Algorithm 3 compute $\chi\left(W_{\varepsilon}^{h}\right)$.

Step 3. Output $\chi(W)=\frac{1}{2} \chi\left(W_{\varepsilon}^{h}\right)$.

Proof of correctness. The correctness of Algorithm 4 follows from 2.20 and the correctness of Algorithm 3

Complexity analysis. The complexity of the algorithm is clearly $(\ell s m d)^{O(m+k)}$ arithmetic operations in $\mathrm{R}\langle\varepsilon\rangle$ from the complexity analysis of Algorithm 3 Moreover the maximum degree in $\varepsilon$ is bounded by $(\ell m d)^{O(m+k)}$. Finally, the complexity of the algorithm is $(\ell s m d)^{O(m+k)}$ arithmetic operations in R.

3.2.3. The case of a $\mathcal{Q} \cup \mathcal{P}$-closed semi-algebraic set. Since we want to deal with a general $\mathcal{Q} \cup \mathcal{P}$-closed semi-algebraic set, we shall need a property similar to Corollary 3.3 in a context where all the sets considered are closed and bounded.

We need a few preliminary definitions and results. Let $\mathcal{Q}=\left\{Q_{1}, \ldots, Q_{m}\right\}$ and

$$
0<\varepsilon_{m} \ll \cdots \ll \varepsilon_{1} \ll \varepsilon_{0} \ll 1
$$

be infinitesimals. For every $j \in[m]=\{1, \ldots, m\}$, denote $\mathrm{R}_{j}=\mathrm{R}\left\langle\varepsilon_{0}, \ldots, \varepsilon_{j}\right\rangle$. Let

$$
\begin{array}{ll}
\Psi_{i}^{0}=\left(Q_{i}=0\right), & \\
\Psi_{i}^{1}=\left(Q_{i} \geq \varepsilon_{i}\right), & \Psi_{i}^{-1}=\left(Q_{i} \leq-\varepsilon_{i}\right), \\
\Psi_{i}^{2}=\left(Q_{i}=\varepsilon_{i}\right), & \Psi_{i}^{-2}=\left(Q_{i}=-\varepsilon_{i}\right) .
\end{array}
$$

The following lemma plays a role similar to Corollary 3.3

Lemma 3.4. Let $S$ be a $\mathcal{Q} \cup \mathcal{P}$-closed bounded semi-algebraic set. For every $j \in[\mathrm{m}]$,

$\chi(S)=\chi\left(\mathcal{R}\left(\Psi_{j}^{0}, S\right)\right)+\chi\left(\mathcal{R}\left(\Psi_{j}^{1}, S\right)\right)+\chi\left(\mathcal{R}\left(\Psi_{j}^{-1}, S\right)\right)-\chi\left(\mathcal{R}\left(\Psi_{j}^{2}, S\right)\right)-\chi\left(\mathcal{R}\left(\Psi_{j}^{-2}, S\right)\right)$.

Proof. This follows from the additivity property of the Euler-Poincaré characteristic, and the fact that

$$
\chi\left(\mathcal{R}\left(\Psi_{j}^{0}, S\right)\right)=\chi\left(\left\{(x, y) \in S \mid-\varepsilon_{j} \leq Q_{j}(x, y) \leq \varepsilon_{j}\right\}\right),
$$

since $\mathcal{R}\left(\Psi_{j}^{0}, S\right)$ is a deformation retract of $\left\{(x, y) \in S \mid-\varepsilon_{j} \leq Q_{j}(x, y) \leq \varepsilon_{j}\right\}$.

We define $\Sigma_{m}=\{-2,-1,0,1,2\}^{[m]}$. Given $\rho \in \Sigma_{m}$ we define

$$
\mathcal{R}(\rho, S)=\left\{(x, y) \in \operatorname{Ext}\left(S, \mathrm{R}_{m}\right) \mid \bigwedge_{i=1}^{m} \Psi_{i}^{\rho(i)}(x, y)\right\} .
$$

For any $\rho \in \Sigma_{m}$ and $\sigma$ a weak sign condition on $\mathcal{Q} \cup \mathcal{P}$, we say that $\rho \prec \sigma$ if for each $i \in[m], \operatorname{sign}(\rho(i)) \in \sigma\left(Q_{i}\right)$ and $\mathrm{R}(\sigma) \subset S$.

Notice that an alternative description of $\mathcal{R}(\rho, S)$ is given by

$$
\mathcal{R}(\rho, S)=\left\{(x, y) \in \mathrm{R}_{m}^{\ell+k} \mid \bigwedge_{i=1}^{m} \Psi_{i}^{\rho(i)}(x, y) \wedge\left(\bigvee_{\rho \prec \sigma} \bigwedge_{P \in \mathcal{P}}(\operatorname{sign}(P(x) \in \sigma(P)))\right\}\right.
$$


Algorithm 5 (Euler-Poincaré, the general case).

\section{Input:}

- a family of polynomials, $\mathcal{Q}=\left\{Q_{1}, \ldots, Q_{m}\right\} \subset \mathrm{R}\left[Y_{1}, \ldots, Y_{\ell}, X_{1}, \ldots, X_{k}\right]$, with $\operatorname{deg}_{Y}(Q) \leq 2, \operatorname{deg}_{X}(Q) \leq d$,

- another family of polynomials, $\mathcal{P} \subset \mathrm{R}\left[X_{1}, \ldots, X_{k}\right]$, with $\operatorname{deg}_{X}(P) \leq d, P \in \mathcal{P}$, $\#(\mathcal{P})=s$,

- a $\mathcal{Q} \cup \mathcal{P}$-closed formula defining a $\mathcal{Q} \cup \mathcal{P}$-closed semi-algebraic set $S$.

Output: the Euler-Poincaré characteristic $\chi(S)$.

\section{Procedure:}

Step 1. Define $Q_{0}=\varepsilon_{0}^{2}\left(Y_{1}^{2}+\cdots+Y_{\ell}^{2}\right)-1, P_{0}=\varepsilon_{0}^{2}\left(X_{1}^{2}+\cdots+X_{k}^{2}\right)-1$. Replace $\mathcal{P}$ by $\mathcal{P} \cup\left\{P_{0}\right\}$ and $S$ by $\mathcal{R}(S, \mathrm{R}\langle\varepsilon\rangle) \cap\left(\mathcal{R}\left(Q_{0} \leq 0\right) \times \mathcal{R}\left(P_{0} \leq 0\right)\right)$.

Step 2. For every generalized sign condition $\rho \in \Sigma_{m}$ compute $\chi(\mathcal{R}(\rho, S))$ using 3.2 and Algorithm 4 .

Step 3. Denoting $n(\rho)=\#(\{i \in[m]|| \rho(i) \mid=2\})$, output

$$
\chi(S)=\sum_{\rho}(-1)^{n(\rho)} \chi(\mathcal{R}(\rho, S)) .
$$

Proof of correctness. It follows from the local conic structure of semi-algebraic sets at infinity [15, Theorem 9.3.6] that replacing $S$ by $\mathcal{R}(S, \mathrm{R}\langle\varepsilon\rangle) \cap\left(\mathcal{R}\left(Q_{0} \leq 0\right) \times \mathcal{R}\left(P_{0} \leq 0\right)\right)$ does not modify the Euler-Poincaré characteristic. The proof can now be reduced to the following lemma.

Lemma 3.5. Let $S$ be a $\mathcal{Q} \cup \mathcal{P}$-closed and bounded semi-algebraic set. Denoting $n(\rho)=$ $\#(\{i \in[m]|| \rho(i) \mid=2\})$ for $\rho \in \Sigma_{m}$, we have

$$
\chi(S)=\sum_{\rho \in \Sigma_{m}}(-1)^{n(\rho)} \chi(\mathcal{R}(\rho, S)) .
$$

Proof. The proof is by induction on $m$. The induction hypothesis $H_{j}$ states that denoting $n(\rho)=\#(\{i \in[j]|| \rho(i) \mid=2\})$ for $\rho \in \Sigma_{j}$, we have

$$
\chi(S)=\sum_{\rho \in \Sigma_{j}}(-1)^{n(\rho)} \chi(\mathcal{R}(\rho, S)) .
$$

The base case $H_{1}$ is exactly Lemma 3.4 applied to $S$. Suppose now that $H_{j-1}$ holds for some $1<j \leq m$, i.e.

$$
\chi(S)=\sum_{\rho \in \Sigma_{j-1}}(-1)^{n(\rho)} \chi(\mathcal{R}(\rho, S))
$$

and let us prove $H_{j}$. Define $\mathcal{Q}_{j}=\mathcal{Q} \cup\left\{Q_{i} \pm \varepsilon_{i} \mid i=1, \ldots, j\right\}$.

For every $\rho \in \Sigma_{j-1}, \mathcal{R}(\rho, S)$ is a $\mathcal{Q}_{j-1} \cup \mathcal{P}$-closed semi-algebraic set. Denoting by $\rho_{i} \in \Sigma_{j}$, for $\rho \in \Sigma_{j-1}, i \in\{-2,-1,0,1,2\}$, the generalized sign condition defined by 
$\rho_{i}(u)=\rho(u), u=1, \ldots, j-1, \rho_{i}(j)=i$, notice that $\mathcal{R}\left(\rho_{i}, S\right)=\mathcal{R}\left(\Psi_{j}^{i}, \mathcal{R}(\rho, S)\right)$. Applying Lemma 3.4 to $\mathcal{R}(\rho, S)$, we obtain

$$
\begin{aligned}
\chi(\mathcal{R}(\rho, S))= & \chi\left(\mathcal{R}\left(\rho_{0}, S\right)\right)+\chi\left(\mathcal{R}\left(\rho_{1}, S\right)\right)+\chi\left(\mathcal{R}\left(\rho_{-1}, S\right)\right) \\
& -\chi\left(\mathcal{R}\left(\rho_{2}, S\right)\right)-\chi\left(\mathcal{R}\left(\rho_{-2}, S\right)\right) .
\end{aligned}
$$

Substituting each $\chi(\mathcal{R}(\rho, S))$ by its value in 3.3 one gets $H_{j}$, since every element of $\Sigma_{j}$ is of the form $\rho_{i}$ for some $\rho \in \Sigma_{j-1}, i \in\{-2,-1,0,1,2\}$.

Complexity analysis. There are $5^{m}$ calls to Algorithm 4 . The complexity of the algorithm is clearly $(\ell s m d){ }^{O(m+k)}$ arithmetic operations in $\mathrm{R}_{m}$ from the complexity analysis of Algorithm 4. Moreover the maximum degree in $\varepsilon_{0}, \ldots, \varepsilon_{m}$ is bounded by $(\ell m d)^{O(m+k)}$. Finally, the complexity of the algorithm is $(\ell s m d) O(m(m+k))$ arithmetic operations in R.

Proof of Theorem 1.9 The proof of correctness and the complexity analysis of Algorithm 5 also proves Theorem 1.9 .

Acknowledgments. The first author was supported in part by an NSF grant CCF-0634907. The second author was supported by an NTU startup grant. Part of this work was done when the authors were visiting the Institute of Mathematics and Its Application, Minneapolis, IRMAR (Rennes) and the Nanyang Technological University, Singapore.

\section{References}

[1] Agrachev, A. A.: Topology of quadratic maps and Hessians of smooth maps. In: Algebra, Topology, Geometry, Itogi Nauki i Tekhniki, vol. 26, Akad. Nauk SSSR, Vsesoyuz. Inst. Nauchn. Tekhn. Inform.,VINITI, Moscow, 85-124 (1988) (in Russian); English transl. J. Soviet Math. 49, 990-1013 (1990) Zbl 0719.58006 MR 0978394

[2] Barvinok, A. I.: Feasibility testing for systems of real quadratic equations. Discrete Comput. Geom. 10, 1-13 (1993) Zbl 0812.12006 MR 1215319

[3] Barvinok, A. I.: On the Betti numbers of semialgebraic sets defined by few quadratic inequalities. Math. Z. 225, 231-244 (1997) Zbl 0919.14034 MR 1464928

[4] Basu, S.: On bounding the Betti numbers and computing the Euler characteristic of semialgebraic sets. Discrete Comput. Geom. 22, 1-18 (1999) Zbl 0973.14033 MR 1692627

[5] Basu, S.: Algorithmic semi-algebraic geometry and topology—recent progress and open problems. In: Surveys on Discrete and Computational Geometry: Twenty Years Later, Contemp. Math. 453, Amer. Math. Soc., 139-212 (2008) Zbl 1145.14044 MR 2405681

[6] Basu, S.: Computing the top few Betti numbers of semi-algebraic sets defined by quadratic inequalities in polynomial time. Found. Comput. Math. 8, 45-80 (2008) Zbl 1141.14034 MR 2403530

[7] Basu, S.: Errata for "Computing the top few Betti numbers of semi-algebraic sets defined by quadratic inequalities in polynomial time”. Found. Comput. Math. 8, 81-95 (2008) Zbl 1141.14034 MR 2403531

[8] Basu, S.: Computing the first few Betti numbers of semi-algebraic sets in single exponential time. J. Symbolic Comput. 41, 1125-1154 (2006) Zbl 1126.14065 MR 2262087

[9] Basu, S.: Efficient algorithm for computing the Euler-Poincaré characteristic of a semialgebraic set defined by few quadratic inequalities. Comput. Complexity 15, 236-251 (2006) Zbl 1103.14032 MR 2268404 
[10] Basu, S., Kettner, M.: A sharper estimate on the Betti numbers of sets defined by quadratic inequalities. Discrete Comput. Geom. 39, 734-746 (2008) Zbl pre05313105 MR 2413156

[11] Basu, S., V. Pasechnik, D., Roy, M.-F.: Computing the Betti numbers of semi-algebraic sets defined by partly quadratic systems of polynomials. J. Algebra 321, 2206-2229 (2009) Zbl 1171.14040 MR 2501518

[12] Basu, S., Pollack, R., Roy, M.-F.: Algorithms in Real Algebraic Geometry. 2nd ed., Algorithms Comput. Math. 10, Springer, Berlin (2006) Zbl 1102.14041 2248869

[13] Basu, S., Pollack, R., Roy, M.-F.: Computing the first Betti number of a semi-algebraic set. Found. Comput. Math. 8, 97-136 (2008) Zbl 1142.14036 MR 2403532

[14] Basu, S., Pollack, R., Roy, M.-F.: On the Betti numbers of sign conditions. Proc. Amer. Math. Soc. 133, 965-974 (2005) Zbl 1080.14068 MR 2117195

[15] Bochnak, J., Coste, M., Roy, M.-F.: Géométrie algébrique réelle. Ergeb. Math. Grenzgeb. 12, Springer, Berlin (1987) Zbl 0633.14016 MR 0949442

[16] Chern, S. S., Spanier, E.: The homology structure of sphere bundles. Proc. Nat. Acad. Sci. U.S.A. 36, 248-255 (1950) Zbl 0035.38803 MR 0035994

[17] Gabrielov, A., Vorobjov, N.: Betti numbers of semialgebraic sets defined by quantifier-free formulae. Discrete Comput. Geom. 33, 395-401 (2005) Zbl $1073.14072 \mid$ MR 2121987

[18] Grigoriev, D., Pasechnik, D.: On Betti numbers of semi-algebraic sets over quadratic maps. Manuscript, 2004.

[19] Grigoriev, D., Pasechnik, D. V.: Polynomial-time computing over quadratic maps. I. Sampling in real algebraic sets. Comput. Complexity 14, 20-52 (2005) Zbl 1082.14065 MR 2134044

[20] Hatcher, A.: Algebraic Topology. Cambridge Univ. Press, Cambridge (2002) Zbl 1044.55001 MR 1867354

[21] Milnor, J.: On the Betti numbers of real varieties. Proc. Amer. Math. Soc. 15, 275-280 (1964) Zbl 0123.38302 MR 0161339

[22] Petrovskiǔ, I. G., Oleŭnik, O. A.: On the topology of real algebraic surfaces. Izv. Akad. Nauk SSSR Ser. Mat. 13, 389-402 (1949) Zbl 0035.10204 MR 0034600

[23] Smale, S.: A Vietoris mapping theorem for homotopy. Proc. Amer. Math. Soc. 8, 604-610 (1957) Zbl 0089.39003 MR 0087106

[24] Thom, R.: Sur l'homologie des variétés algébriques réelles. In: Differential and Combinatorial Topology (A Symposium in Honor of Marston Morse), Princeton Univ. Press, Princeton, NJ, 255-265 (1965) Zbl 0137.42503 MR 0200942 